\title{
The science case and mission concept for the Single Aperture Far- Infrared (SAFIR) Observatory
}

Daniel F. Lester, Dominic J. Benford, Andrew Blain, Charles M. Bradford, Mark Dragovan, et al.

Daniel F. Lester, Dominic J. Benford, Andrew Blain, Charles M. Bradford, Mark Dragovan, William Langer, Charles R. Lawrence, David T. Leisawitz, John C. Mather, Samuel Harvey Moseley, Lee G. Mundy, George H. Rieke, Gordon Stacey, Harold W. Yorke, Erick T. Young, "The science case and mission concept for the Single Aperture Far-Infrared (SAFIR) Observatory," Proc. SPIE 5487, Optical, Infrared, and Millimeter Space Telescopes, (12 October 2004); doi: 10.1117/12.551335

Event: SPIE Astronomical Telescopes + Instrumentation, 2004, Glasgow, United Kingdom 


\title{
The Science Case and Mission Concept for the Single Aperture Far-Infrared (SAFIR) Observatory
}

\author{
Daniel Lester*,a, Dominic Benford ${ }^{\mathrm{b}}$, Andrew Blain ${ }^{\mathrm{c}}$, C. Matt Bradford ${ }^{\mathrm{d}}$, Mark Dragovan ${ }^{\mathrm{d}}$, William \\ Langer $^{\mathrm{d}}$, Charles Lawrence ${ }^{\mathrm{d}}$, David Leisawitz ${ }^{\mathrm{b}}$, John Mather ${ }^{\mathrm{b}}$, S. Harvey Moseley ${ }^{\mathrm{b}}$, Lee Mundy ${ }^{\mathrm{e}}$, \\ George Rieke ${ }^{\mathrm{f}}$, Gordon Stacey ${ }^{\mathrm{g}}$, Harold Yorke ${ }^{\mathrm{d}}$, Erick Young ${ }^{\mathrm{f}}$ \\ ${ }^{\text {a } D e p a r t m e n t ~ o f ~ A s t r o n o m y ~ a n d ~ M c D o n a l d ~ O b s e r v a t o r y, ~ U n i v e r s i t y ~ o f ~ T e x a s, ~ A u s t i n ~ T X ~}$ \\ ${ }^{\mathrm{b}} \mathrm{Lab}$ for Astronomy and Solar Physics, Infrared Astrophysics Branch, \\ NASA Goddard Space Flight Center, Greenbelt MD \\ ${ }^{c}$ Department of Astronomy, California Institute of Technology, Pasadena CA \\ ${ }^{\mathrm{d}}$ Division of Earth and Space Sciences Jet Propulsion Laboratory, \\ California Institute of Technology, Pasadena CA \\ ${ }^{\mathrm{e}}$ Department of Astronomy, University of Maryland, College Park MD \\ ${ }^{\mathrm{f}}$ Department of Astronomy and Steward Observatory, University of Arizona, Tucson AZ \\ ${ }^{\mathrm{g}}$ Department of Astronomy, Cornell University, Ithaca NY
}

\begin{abstract}
SAFIR is a large ( $10 \mathrm{~m}$-class), cold $(4-10 \mathrm{~K})$ space telescope for wavelengths between 20 microns and $1 \mathrm{~mm}$. It will provide sensitivity a factor of a hundred or more greater than that of Spitzer and Herschel, leveraging their capabilities and building on their scientific legacies. Covering this scientifically critical wavelength regime, it will complement the expected wavelength performance of the future flagship endeavors JWST and ALMA. This vision mission will probe the origin of stars and galaxies in the early universe, and explore the formation of solar systems around nearby young stars. Endorsed as a priority by the Decadal Study and successive OSS roadmaps, SAFIR represents a huge science need that is matched by promising and innovative technologies that will allow us to satisfy it. In exercising those technologies it will create the path for future infrared missions. This paper reviews the scientific goals of the mission and promising approaches for its architecture, and considers remaining technological hurdles. We review how SAFIR responds to the scientific challenges in the OSS Strategic Plan, and how the observatory can be brought within technological reach.
\end{abstract}

\section{INTRODUCTION}

\subsection{Summary}

A $10 \mathrm{~m}$-class cold telescope equipped for infrared science, and envisioned to begin development late in the decade, the Single Aperture Far Infrared Observatory (SAFIR) is truly the next generation facility-class infrared telescope. With a light collecting power two orders of magnitude larger than any existing infrared telescope, and new generation infrared arrays at the focal plane that dramatically multiply its observing efficiency and effectively harness a spatial resolution that rivals that of ground-based optical telescopes, SAFIR offers great promise. The National Academy of Sciences Decadal Review identified SAFIR as a high priority successor to Spitzer, and an important scientific partner to JWST and ALMA. Responding to the science community, the telescope has been identified as a key element in roadmaps of the NASA Office of Space Science. SAFIR represents a convergence of fundamental science need coupled with emerging technological feasibility.

\subsection{Background of the Vision Mission Study}

The SAFIR concept embraces that of other large infrared telescopes that have recently been proposed, such as FAIR and DART. NASA, industry, and the greater science community have invested strongly in the concept for several years. This investment has culminated in the selection of SAFIR for a Vision Mission study that is now in progress. Our study team includes formal members from four NASA centers, with GSFC and JPL providing leadership on science trades and

*dfl@astro.as.utexas.edu; phone 1-512-471-3442; fax 1-512-471-6016 http://www.as.utexas.edu/ 
engineering requirements, MSFC evaluating primary mirror materials, and JSC exploring human-aided deployment options. Team members include Ball Aerospace, Boeing, Lockheed-Martin, and Northup-Grumman, bringing a wide range of state-of-art technical and engineering expertise to the program. It includes key members of the Spitzer, Herschel and SOFIA programs. As a cold, large aperture far infrared space telescope, SAFIR is viewed by the far infrared and submillimeter community plan as a consensus priority (see the Community Plan for Far IR-Infrared and Submillimeter Astronomy, "Second Workshop on New Concepts for Far-Infrared/Submillimeter Space Astronomy," March 2002, College Park, Maryland) and a critical part of the scientific and technical path that will lead to highly capable far infrared interferometers such as SPECS. The process that has led to this consensus represents true partnership between agency and community. A powerful tool for studying the universe from the youngest, most distant galaxies to the details of star and planet formation nearby, SAFIR addresses four enterprise objectives of the NASA Office of Space Science.

With performance baselined for the 20-800 micron wavelength range that is termed the mid- and far-infrared through submillimeter, SAFIR is poised to bridge the 1.5 decades of the spectrum between JWST and ALMA. It will enhance the return on these facilities by unifying the disparate views they will give us on the cosmos. This broad part of the spectrum, which includes the energy peak of galaxies and newly forming stars, critical cooling lines for interstellar clouds, and light molecular tracers, has been chronically unexplored due to the opacity of the Earth's atmosphere across the entire spectral region. Making the job challenging is that at these long wavelengths, spatial resolution that is required to distinguish target sources requires large apertures, and the highest sensitivity requires operation at the lowest temperatures.

\subsection{SAFIR in the Context of Infrared Astronomical Investments}

The field of far-infrared astronomy began fifty years ago, with balloon, aircraft, and sounding rocket explorations of the astronomical sky (Soifer and Pipher 1978). The low resolution all-sky survey completed by the $57 \mathrm{~cm}$ liquid heliumcooled IRAS telescope (Beichman et al 1988) brought infrared astronomy to the fore, highlighting the richness of the sky at these wavelengths. Coupled with higher resolution studies by the balloon and airborne observatory programs that offered opportunities to near-space conditions with a wide variety of astronomical imagers and spectrographs, the field rapidly matured. These programs included the $91 \mathrm{~cm}$ Kuiper Airborne Observatory (KAO). While its sensitivity was limited by the stratospheric ambient temperature and atmospheric absorption, the $\mathrm{KAO}$ allowed regular access to the infrared sky, a strategic blessing for a field in which sensor technology was rapidly growing in capability and sophistication. From 1995 to 1998, the Infrared Astronomical Satellite (ISO) of ESA (Kessler et al. 1996) operated as a cryogenic infrared space observatory. While ISO had a primary mirror size that was comparable $(60 \mathrm{~cm})$ to that of IRAS, the improved and diffraction-limited pixels took full advantage of the spatial resolution that was offered. ISO offered a pointed observing mode, and a suite of instruments including high resolution spectrometers. Most recently, the success of the Spitzer Space Telescope (formerly SIRTF - see Gallagher, Irace, and Werner 2003) has been a crowning achievement for the infrared community. With a $0.85 \mathrm{~m}$ aperture, and a sophisticated suite of instruments that include state-of-art detectors, this cryogenic facility-class telescope is dramatically improving our view of the far infrared sky. The Japanese mission Astro-F, scheduled to be launched in 2005, is similarly sized to IRAS, but will carry out a far infrared survey with a focal plane that is dramatically improved over that of IRAS in format and sensitivity.

With the ISOPHOT and MIPS cameras (on ISO and Spitzer respectively) reaching out to the photoconductor limit near $200 \mu \mathrm{m}$, the full mid and far infrared will have been explored from space. Such efforts for submillimeter bands inaccessible from the ground are still in relative infancy. The KAO, which provided some capability here, was a powerful community asset and training ground until it was decommissioned in 1996 to invest in development of the 2.5 $\mathrm{m}$ Stratospheric Observatory for Infrared Astronomy (SOFIA). SOFIA should see the first research flights within a year. It will be operated by NASA, but is being developed jointly by NASA and DLR, and will serve U.S. and German science communities. While SOFIA will offer continual access to new instruments, and in doing so greatly stimulate technology development for infrared and submillimeter efforts in general, it is a warm telescope, and will not see most of the sources that Spitzer detects. The $3.5 \mathrm{~m}$ Herschel Space Observatory scheduled for launch in 2007 is also relatively warm and has a fixed instrument complement. Nevertheless, Herschel will avoid the residual telluric interference that a stratospheric telescope faces.

Ground-based telescopes at extraordinary sites such as the South Pole and the Chajnantor region of the Chilean Atacama desert offer performance in narrow submillimeter spectral bands at wavelengths that may perhaps go shortward of 350 microns. This is in narrow windows, however, and within which very significant opacity remains. Such opacity and 
warm optics compromise the performance of these telescopes compared with cold telescopes in space. That these ground based sites offer near-term opportunities for vastly larger telescopes (e.g. ALMA, APEX, AT-25), however, providing spatial resolution that exceeds that available in the near term from space, a productive scientific partnership is ensured.

\subsection{The SAFIR Vision}

It is with this backdrop that SAFIR is proposed. SAFIR is envisioned as a cold $10 \mathrm{~m}$-class observatory facility developed for operation as early as the middle of the next decade. With an operating temperature near that of Spitzer, it will provide sky background-limited performance. Its much larger aperture will provide spatial resolution that is three times better than Herschel and SOFIA, and ten times better than Spitzer, taking full advantage of the rapid increase in focal plane array formats. SAFIR will offer a tremendous leap in observational capability. For example, the improvement in mapping speed which is proportional to pixels/sensitivity ${ }^{2}$ will be $\sim 10^{6}$ over what is achievable now, with two orders of magnitude more detectors in the focal plane and a point source sensitivity that is two orders of magnitude higher than Spitzer. While there are several ways that SAFIR could be implemented, the architecture now being developed for the James Webb Space Telescope (JWST) for launch in 2011 is an existence proof in this regard, since the needs for JWST and SAFIR overlap strongly. SAFIR is somewhat colder and larger than JWST, but requires a wavefront accuracy that is 10-20 times lower. With Spitzer just launched, and SOFIA and Herschel just ahead, it is clear that the scientific and technical trajectory that they define points directly at the need for an observatory like SAFIR. This was recognized by the Decadal panel of the National Academy of Sciences and in the strategic planning efforts of the NASA Office of Space Science. Developing large, cold, infrared telescopes in space is a key to achieving more scientifically ambitious missions, such as the SPECS interferometer, and SAFIR should be seen programmatically as part of a broadly focused mission line. There is a high level of interest in space observatories at these wavelengths, and opportunities for international collaborations on SAFIR can be considered timely.

\section{SCIENCE GOALS AND SIGNIFICANCE OF SAFIR}

We review below several primary science goals of SAFIR to illustrate the breadth and depth of the mission science. These goals link directly with identified priorities in the strategic plan for the NASA Office of Space Science.

\subsection{Making Primordial Stars}

Among the most exciting results of the Wilkinson Microwave Anisotropy Probe (WMAP) is the measurement of an optical depth, and the inference of star formation at z 20 . Reionization of the primordial gas by UV photons from these very first stars is a huge milestone in cosmic evolution, often called the "end of the dark ages." These first stars make the first heavy elements (C, N, O, Fe, etc) - "metals" - which profoundly influence subsequent star, galaxy, and planet formation. Probing reionization will be a key program for future astrophysics missions. Important spectral lines from the formation of the very first $(\mathrm{H}, \mathrm{He})$ stars are emitted at restframe near and mid-IR wavelengths and will be observed in the mid-IR to submillimeter, where SAFIR's huge sensitivity offers excellent discovery potential. A major thrust for SAFIR will be in studying the evolution of primordial stars.

Several recent important results pertain: (1) As the stars form, collapsing clouds are cooled primarily by molecular hydrogen transitions at 8-30 microns rest wavelength. Redshifted, such lines are in the far-IR and submillimeter. During collapse, a single such protostar can radiate $10-100 \mathrm{~L}_{\mathrm{o}}$ in each of the handful of these rotational $\mathrm{H}_{2}$ lines. It is estimated that a large $\left(10^{11} \mathrm{M}_{\mathrm{o}}\right)$ protogalaxy could harbor as many as $10^{4}$ such protostars at a given time over its $10^{7} \mathrm{yr}$ dynamical lifetime. Such a collection could be detectable by SAFIR. (2) The supernova explosion from an initial massive star could produce overdense shocks in the ISM and reform $\mathrm{H}_{2}$, which can then radiate as much as $10 \%$ of the $\sim 10^{53} \mathrm{ergs}$ it releases. Such an object would outshine the rest of the host galaxy and be detectable with SAFIR.

\subsection{When the Universe Made Galaxies}

It is now understood that the far-IR and submillimeter sky is strewn with cosmological sources. The powerful infrared background is dominated by sources unresolved by existing missions. They were only partly resolved, for example, by ISO. These sources generally have very faint optical counterparts, and are thought to be heavily obscured young galaxies near the epoch of most intense star and galaxy formation. We know little about these except that they are blue in the submillimeter, with redshifted luminosity output that peaks at several hundred microns. SAFIR will resolve these galaxies, image them at their spectral peaks, and measure their photometric and spectroscopic redshifts, luminosities, physical conditions and star formation rates. With the high spatial resolution that SAFIR offers, we will be able to probe 
the importance of galaxy collisions and mergers in these first generation galaxies. JWST should detect these first galaxies, but SAFIR will tell us why they hide in the optical. In this way, SAFIR will trace back star formation in the universe in an unbiased way past the peak of galaxy formation to $z>5$. The history of star formation in the universe is closely related to the buildup of heavy elements, and the chemical enrichment of the gas in the universe is of critical importance in feedback on the cooling rates of interstellar gas. For example, once carbon is synthesized, the 158 micron line of $\mathrm{C}^{+}$is a powerful coolant of the warm gas out of which stars form. Using SAFIR, we will be able to image the dominant phases of dustembedded star formation and nuclear activity throughout the universe. Our study will consider SAFIR sensitivity goals on the basis of model stellar populations at this redshift, taking into account the latest understanding of source luminosity functions and K-corrections from Spitzer.

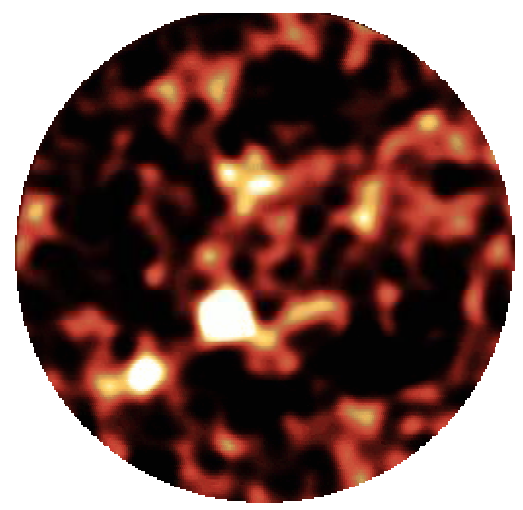

The submillimeter galaxies that account for all the structure in this 3' HDF JCMT 850 micron map will be dramatically brighter in the far-IR. SAFIR will map them easily, and analyze each of them spectroscopically. These sources are distributed quite differently than even the faintest and most distant galaxies detected in the Hubble Deep Field optical survey, and must thus represent a completely different class of galaxy in the early universe.

\subsection{Seeing into the Heart of Galaxies}

While on average in the local universe, somewhat less than half of the energy emerges at far infrared wavelengths, such emission accounts for well more than half the background. This is because the primary luminosity sources in galaxies are often obscured by dust, and these emitters get relatively more important at higher redshift. The far infrared is not susceptible to extinction, and a key thrust for SAFIR will be to directly observe the obscured regions of nuclear accretion. Infrared spectroscopy offers excellent tools for measuring gas mass, density, and temperature as well as ionizing radiation hardness. Two fundamental questions can be addressed about these galactic nuclei.

One is the energy source in Ultraluminous Infrared Galaxies (ULIGs). Are these astonishingly IR-bright $\left(>10^{12} \mathrm{~L}_{0}\right)$ galaxies powered by accretion onto massive nuclear black holes, by superstarbursts, or by a combination of the two? FarIR fine-structure lines that probe UV radiation hardness will provide answers to this. Diffraction-limited with a large aperture, SAFIR will resolve local ULIGs at their spectral peaks. Initial estimates suggest that SAFIR will be able to observe ULIGs out to $\mathrm{z} \sim 10$, well before the peak in their star formation activity,

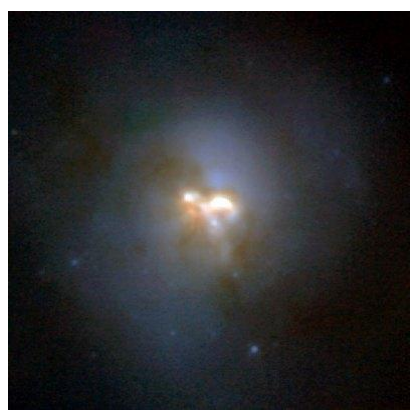

Arp220 - a well known ULIG. The optical appearance of Arp 220 (shown here) is strongly influenced by dust obscuration, and the infrared view allows us to better measure the intrinsic properties of this source. While Arp 220 is relatively nearby and a remarkable constituent of the local universe, it is more representative of galaxy populations in the early universe, when galaxy-building was rampant.

Current theories hold that the two types of Seyfert galaxies, Seyfert Is and IIs, are the same type of object - a central accretion engine surrounded by a dense torus of molecular gas. Is this the result of a different viewing geometry Seyfert IIs being viewed through the torus, and Seyfert Is being viewed unobscured along the axis of the torus? SAFIR can test this second question in two ways: 1) Spectroscopy of far-IR fine structure lines from the ionized medium - if present they will be observable regardless of the torus angle, and 2) Detection of the molecular torus itself in the far-IR rotational transitions of $\mathrm{CO}, \mathrm{OH}, \mathrm{H}_{2} \mathrm{O}$. 

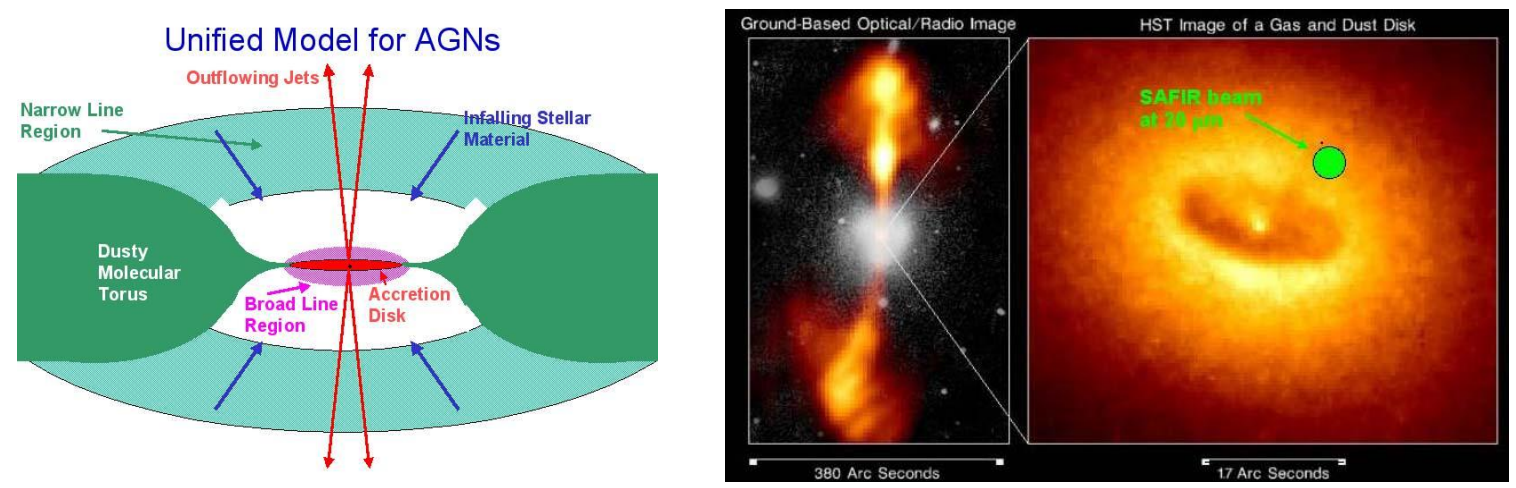

At left, a cartoon showing the unified model for active galactic nuclei. This model accounts for the optical and ultraviolet visibility of the central engine of some active galaxies, while in others it is invisible due to line of sight through a dense, opaque accretion disk that powers the source. At right, in the active galaxy NGC4261, the radio image shows dramatic jet structure, which is indicative of an energetic accretion disk. The optical picture shows the illuminated inner edge of the dusty molecular torus with the active nucleus peeking through. The SAFIR beamsize will be well suited to resolving the

torus in this representative galaxy, using the rich diagnostic spectrum available (see below) to explore the torus.

\subsection{SAFIR and the Formation of Stars and Planets}

Except at the earliest epochs, dust plays a prominent role in the formation of stars and planets. It is the principal actor in the thermal evolution and structure of the dense molecular clumps from which they form. During early phases of collapse, dust obscures the emission at short wavelengths, but allows the thermal energy generated during compression to be radiated away, enabling continued contraction. Grain surfaces act as a chemical catalyst for the production of complex molecules, including organics, both prior to and during the star formation process. The result of chemical processing in star forming regions is a richness of molecular species. By studying line shapes, intensities, and excitation, we can extract information on the conditions in these objects, because many ions and molecules do not co-exist but rather trace different regions. Finally, this dust is the raw material for building up planetesimals and comets, from clouds to protostars, Kuiper Belt Objects (KBOs), and ultimately, planets. SAFIR will address several critical questions. How do cloud cores collapse? How does subfragmentation occur, which results in binary and multiple stellar systems? What are the conditions within protostellar disks? When, where, and how frequently do they form planets?

The spatial resolution of SAFIR will allow us to probe the density, dynamics and temperature structure of collapsing cores on critical physical scales. For example, structure on scales of $<100 \mathrm{AU}$ will be accessible for the nearest star forming regions, allowing detailed understanding of fragmentation processes that lead to multiple star systems.

Molecular gas cooling in the inner parts of protostellar clouds is understood to be dominated by high-J rotational lines of $\mathrm{CO}$ and $\mathrm{H}_{2} \mathrm{O}$, and observations of these bright lines by SAFIR will help us track the collapse. SAFIR can harness both gaseous and solid state spectral tracers in making these discoveries.

SAFIR will make direct measurements of fossil debris clouds on Kuiper Belt scales surrounding stars that have already formed solar systems. While present infrared telescopes can barely resolve the morphology of these debris clouds, SAFIR will easily do so. New predictions suggest that the peaks and voids that are seen in these debris clouds are likely the result of quasi-resonances that are set up by the orbits of large planets. SAFIR will use this cloud structure to reveal large planets. Even in relatively cool quiescent debris clouds, SAFIR will spectroscopically characterize ices and minerology, exploring the environs of nearby solar systems on scales that can be compared and contrasted with our own.

\section{UNIQUENESS OF SAFIR}

The unique contributions to be made by SAFIR were captured by the McKee-Taylor Decadal Survey: "The SAFIR Observatory will ... study the relatively unexplored region of the spectrum between 30 and 300 microns. It will enable the study of galaxy formation and the earliest stage of star formation by revealing regions too enshrouded by dust to be studied by NGST, and too warm to be studied effectively with ALMA." We expand on this, emphasizing investigations critical to the OSS Strategic Plan, and how SAFIR contrasts with other missions in this time frame. 
- Since COBE, we know that galaxies generate much of their output in the far-IR. The mid-IR output is dominated by dust heated by young hot stars, so understanding star formation in the main era of galaxy assembly, $z=1-5$, requires study at 30-600 microns. Sensitivity (x100-1000) and angular resolution gains (x10) of SAFIR over Spitzer and Herschel are essential here.

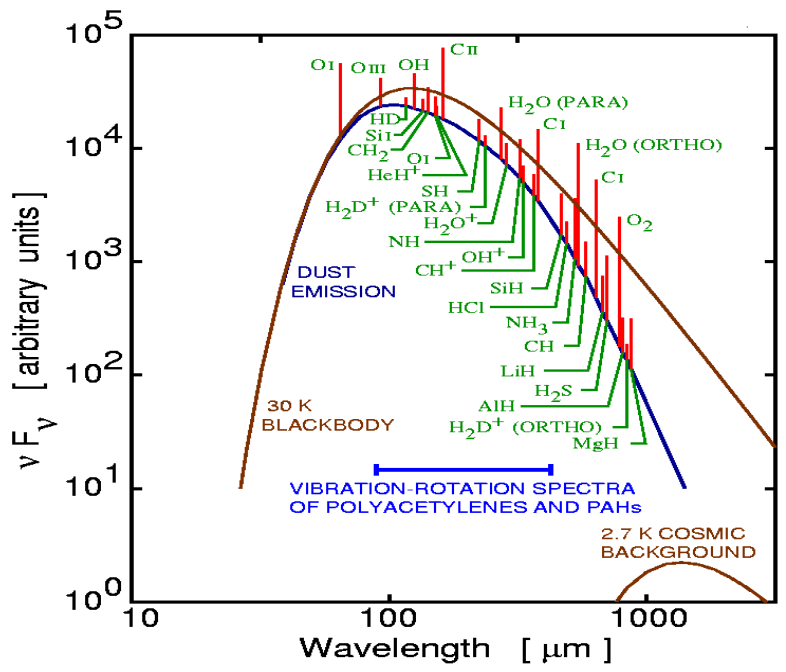

Spectral richness of gas in the far-IR - bright diagnostic lines for SAFIR. These lines, in particular those of $\mathrm{CO}, \mathrm{CI}, \mathrm{C}^{+}$, $\mathrm{H}_{2} \mathrm{O}$ and $\mathrm{OI}$ are powerful coolants for warm interstellar gas, and determine the thermodynamic evolution of collapsing clouds in star formation regions.

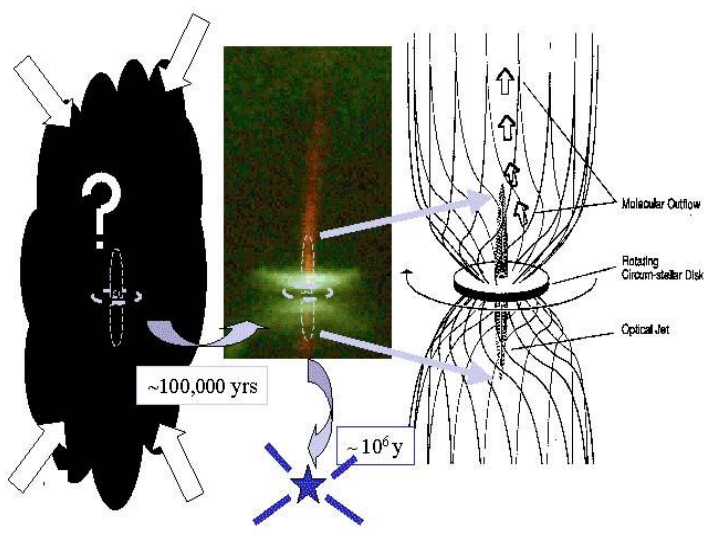

From collapsing cloud to solar system ...

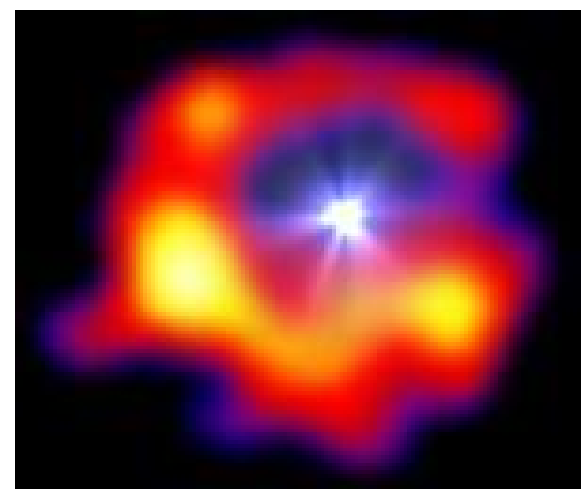

... SAFIR will resolve dusty fossil disks left behind around nearby stars, as in this 850 micron image of Vega from JCMT. Operating near the energy peak, at wavelengths inaccessible from the ground, the spatial resolution will be much higher.

- Far IR fine structure lines (OI, NII, CI, CII, OIII) are among the main coolants of the ISM and can radiate $\sim 1 \%$ of the luminosity of a star forming galaxy. Study of these lines with SAFIR gives new opportunity to watch the buildup of metals at all epochs, which is impossible from the ground. Limited resolution and sensitivity on Herschel and SOFIA prevents such study with those missions, and the wavelength range of Spitzer is inadequate for many of these lines.

- The low lying $\mathrm{H}_{2}$ lines at rest wavelengths 17 and 28.2 microns can reveal pristine gas near pop III stars in the early Universe. Sensitivity and resolution gains over Spitzer, SOFIA, and Herschel are critical, and it cannot be done from the ground.

- The first stages of star and planet formation occur in cold cloud cores that are optically thick at $<2$ microns, dominated by emission in diffuse outer shells at $>500$ microns. SAFIR will offer angular resolution that will let us probe preplanetary disks within these cores. This is unachievable with other IR missions. 
Background-limited, $\mathrm{R}=1000$ at wavelengths $\sim 30-300$ microns, a $10 \mathrm{~m}$ diameter $4 \mathrm{~K} 5 \%$ emissivity SAFIR fills a chasm of sensitivity space in wavelengths between those accessible with JWST and ALMA. SAFIR will be well positioned to attack science defined by Spitzer (formerly SIRTF), Herschel, and SOFIA. Of particular interest for the deepest extraglalactic observations from an observatory site within the inner ecliptic is the background "hole" at 200-300 microns.

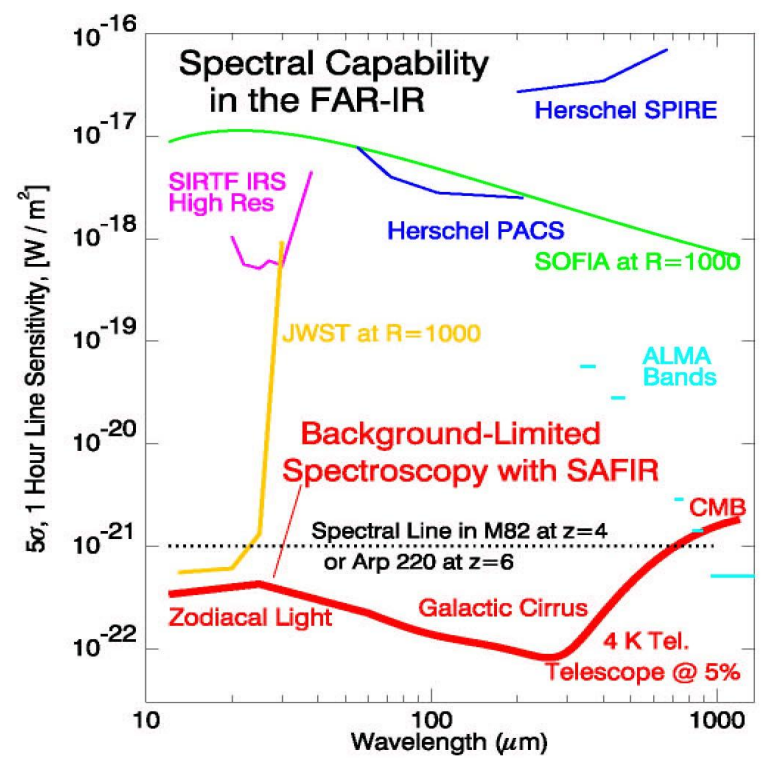

- In cloud cores narrow OI is from infall and broad OI from outflows. $\mathrm{H}_{2} \mathrm{O}$ is a major coolant with a narrow component from a pre-planetary disk and a broad component from accretion. SAFIR has the spatial resolution to exploit these tracers (inaccessible with Spitzer) galaxy-wide much better than Herschel or SOFIA.
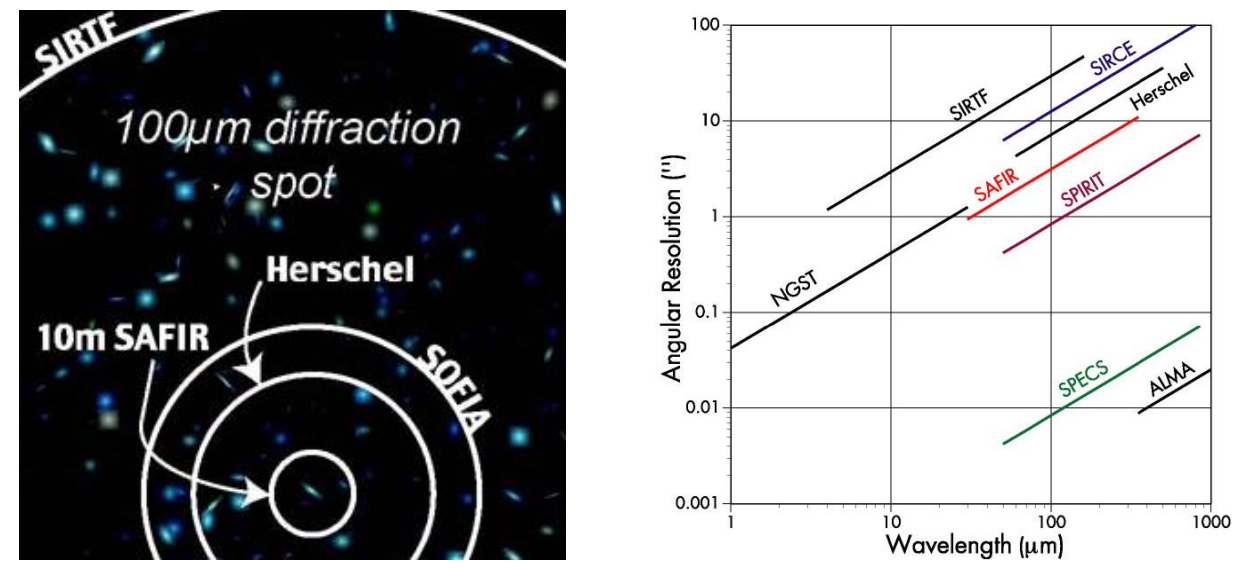

At left, the diffraction spot of planned far-IR observatories on a $15 \times 15$ " simulation of the extragalactic background scaled to the sensitivity of cryogenic telescopes in space. The resolution of SOFIA, which will be for several years the highest available in the far infrared, is shown for comparison. The importance of SAFIR's resolution in avoiding confusion is apparent. At right, the angular resolution of SAFIR is compared to a larger range of infrared and submillimeter telescopes.

- SAFIR has the angular resolution (x10 higher than Spitzer, about 2" at 100 microns) to see structure in debris disks around nearby stars. SAFIR will probe a warmer radial zone not readily accessible by ALMA at longer wavelengths. In addition, SAFIR will be able to study giant planets in the spectral range that dominates their luminosities.

The science goals outlined above translate into the need for line sensitivity of $10^{-21} \mathrm{~W}-\mathrm{m}^{-2}$ and arcsecond angular resolution in the far-IR. To provide the required measurement capabilities we envision SAFIR as a $\sim 10 \mathrm{~m}$ filled aperture telescope that operates at the natural background photon noise limit across the wavelength range from $\sim 20-600$ microns. Background-limited performance is achievable if the optics are cooled to near $4 \mathrm{~K}$ and next-generation detectors, with $\mathrm{NEP}<10^{-19} \mathrm{~W}-\mathrm{Hz}^{-1 / 2}$, are used. 


\section{Technical Approach and Methodology}

\subsection{Matching Architecture to Science Rationale}

Each of the many science goals outlined above requires slightly different aspects of a far-IR observatory and its instruments. A Design Reference Mission (DRM) is being developed that will identify observations required to meet SAFIR's science requirements, and will be used to set technical requirements for wavelength range, sensitivity, spatial resolution, field of view, and science instruments. These lead to requirements in aperture size and temperature, detector sensitivity and number, mirror quality, observatory location, field of regard and associated sunshade geometry, pointing knowledge, data volume for on-board storage, and observing efficiency. Candidate architectures will be evaluated against these requirements, with a minimum aperture size representing about an order of magnitude areal improvement over Herschel.

The mid-IR through submillimeter wavelength range is best for the extinction-independent study of star-ISM interaction, and the required technologies (in-space cryocooling, large telescopes, and sensitive detectors) are just now becoming available. One of the goals for SAFIR is to be able to optimally match JWST at short wavelengths (e.g. 20-40 microns), and ground-based telescopes such as ALMA at long wavelengths (e.g. 350-800 microns). It will match these science needs, which will evolve with the first Spitzer data, with new detector technology. The short wavelength limit will drive mirror quality, alignment and stability needs. At the long wavelength limit, SAFIR offers contiguous spectral coverage that is not available from ground-based telescopes because of narrow atmospheric opacity and thermal emission.

It is important to map the flowdown of science to the raw (e.g. point source) sensitivity for SAFIR. The sensitivity gains of SAFIR over previous generations of IR telescopes are so tremendous that judging from the history of the Great Observatory series of missions our expectations, while seemingly ambitious now, will end up defining a new domain of scientific achievement. The scientific importance of reaching the sky background limit and the importance of source confusion to SAFIR science at different relevant spectral resolutions drive the architecture. Our understanding of these issues will increase with the first observations from Spitzer. For a given-sized telescope, with fixed focal plane spot size, the required point-source sensitivity drives the observatory temperature, and thereby thermal management strategies in general.

At these long wavelengths, the spatial resolution is determined mainly by the size of the observatory. While a defining vision for SAFIR is that of a high resolution far-IR observatory, the specific science goals of SAFIR must be considered in this context, and also the scientific match with observatories operating at nearby wavelengths. Following Stockman et al. who in 1996 conducted a similar study for JWST, image quality (resolution and contrast) is being evaluated from circular and non-circular mirror configurations, and how their different point source profiles impact the science. The impact of image reconstruction on the spatial resolution requirement, and the demands that it makes on observatory and instrument design will bear on the result.

The field of view of SAFIR is a key issue. The scientific importance of large surveys, for which a capability metric is the number of pixels that can be observed simultaneously, is a matter that determines both observatory architecture and detector array requirements. As IR detector array technology is advancing rapidly, huge increases in observatory capability can be foreseen at the focal plane itself. Advances in format size for IR detectors are happening rapidly enough that SAFIR will offer entirely new opportunities in this regard. While the need for broadband imaging seems undeniable, with huge scientific and public outreach appeal, satisfying the broad science requirements for SAFIR will require hard choices in instrument capability. In support of this, we are developing a top-level instrument block diagram for SAFIR that is DRM-compliant, estimating TRL levels of key assembly technologies and recommending a lowest risk approach. The mass, power, and volume of each instrument and of the scientific instrument total package are of interest, as are strategies for managing instrument size and weight. A key issue to be resolved is the importance of $\lambda / \Delta \lambda>30,000$ observations, which would demand investment in heterodyne technologies as well. The scientific priority of a highresolution spectrometer on SAFIR given the capabilities of ALMA, Herschel, and SOFIA is undecided at this time.

\subsection{Architecture and Implementation Approaches}

Our work has shown that several space systems architecture concepts offer SAFIR clear scientific opportunities. Our study will look carefully at these in the context of the DRM, and in the context of technology readiness and trades. It will consider a range of architecture related issues, evaluating structure design, materials, performance analysis, mechanisms 
for deployables including their packaging in a fairing, thermal management (both passive and active), and dynamical issues relating to structures and deployment requirements.

A modified JWST architecture is a baseline starting point for SAFIR trade studies, and has already undergone a GSFC IDC study (July 2002). The JWST includes hexagonal deployable primary and secondary mirrors, a deployable sunshield, and a deployable tower connecting the warm spacecraft bus to the telescope, which resides at Sun-Earth L2, and passively cools to $\sim 40 \mathrm{~K}$. The line of sight can be in any direction in the range of 85-140 degrees from the Sun. Hydrazine thrusters for gyro despin do not contaminate the mirrors because they are on the warm side of the sunshield. The spacecraft bus uses existing technology to provide solar power, command and data handling, on-board memory, and telemetry. Modifications for SAFIR include additional radiative cooling and active cooling using a cascaded system, with an intervening cooled shield at $15 \mathrm{~K}$. This would allow the telescope to reach $\sim 4 \mathrm{~K}$. Additional cryocooling

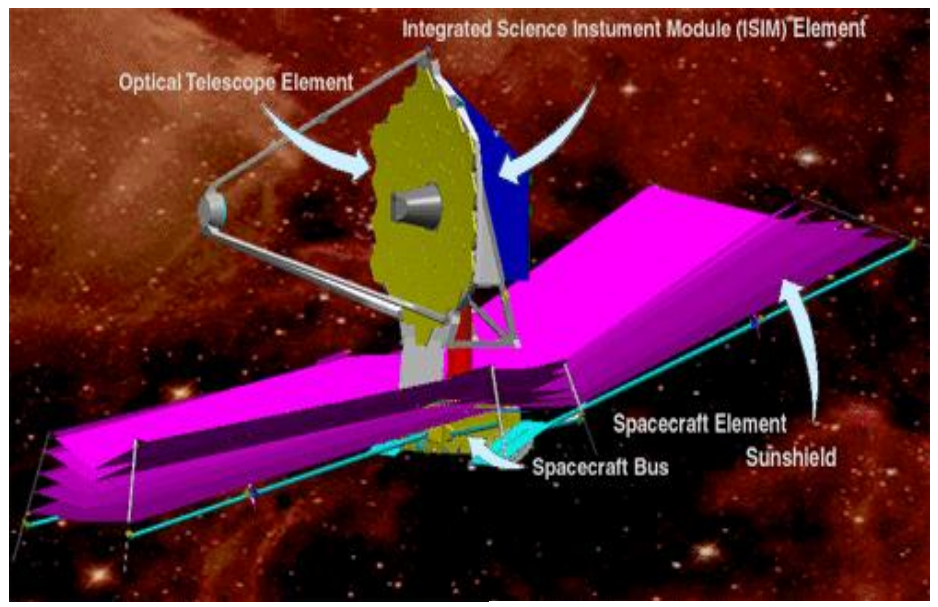

Modified-JWST style SAFIR architecture. In this notional concept, the SAFIR telescope is conceived as a slightly larger and colder version of the JWST point-design, with much lower surface accuracy. Advantages of this architecture are the large investment in JWST observatory technologies, and a well defined starting point for incremental trade studies. Unlike JWST, for which the cooling is entirely passive, SAFIR will probably require active cryocooling in staged layers as shown below.

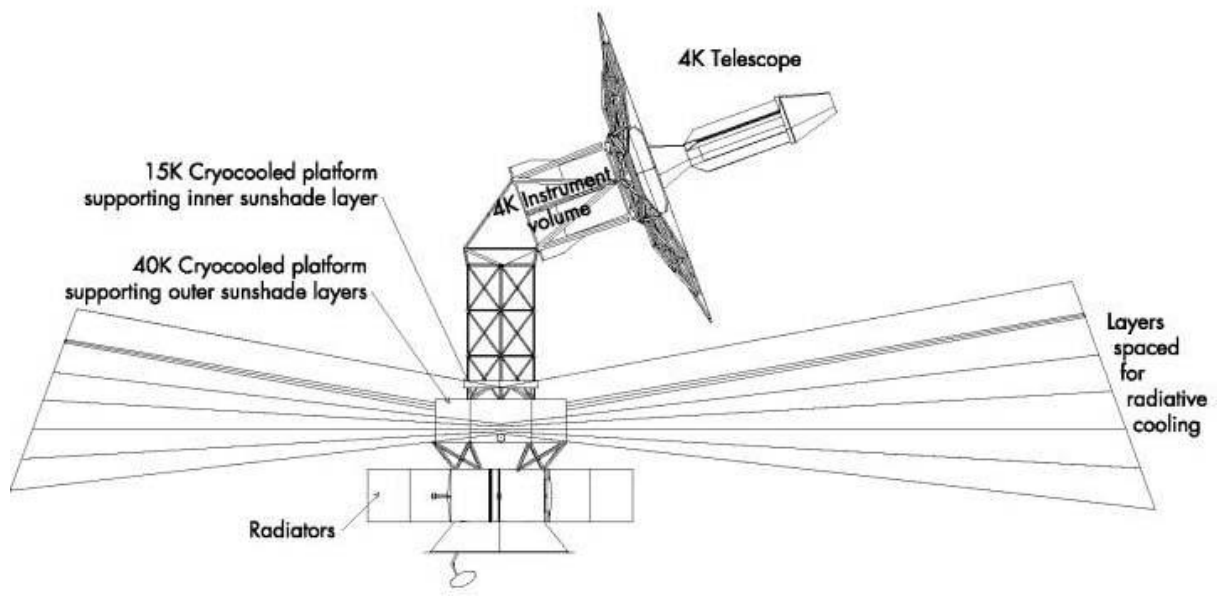

would be used for the sensors themselves.. Since SAFIR is slightly larger than JWST, the design will diverge somewhat, but suitable primary mirror geometries have been suggested that are consistent with packaging in a Delta IV Heavy 19 meter long launch vehicle fairing. Packaging is the major constraint, as this vehicle easily provides the mass-to-L2 capability that SAFIR would need. This general architecture may offer important operating temperature-versus-size trades. While larger than JWST, SAFIR requires a factor of at least ten lower surface accuracy, allowing relaxation of design elements to reduce weight and cost. This concept has been discussed in some detail by Amato et al. (2003).

In support of this baseline design, we are working with Northrop-Grumman, the JWST prime contractor, to study a $4 \mathrm{~K}$ folded mirror JWST-like telescope, an optimally radiatively cooled JWST-like telescope and a much larger passively cooled TPF coronographic concept telescope (but without the coronograph) with card-deck platter-deployment. This will 
include a temperature and emissivity map for each of these three telescopes, cooling rate analysis, and development of parametric relationships between telescope cost, size, and temperature.

A second concept that will be investigated closely is the Dual Anamorphic Reflecting Telescope (DART). The new DART system (see Dragovan, 2002) is dramatically lower in aperture/mass ratio and potentially simpler to deploy than segmented mirror systems. Where a conventional telescope uses a single parabolic mirror, DART uses two ultralight parabolic reflectors of cylindrical symmetry oriented with respect to each other to produce a point focus. These mirrors may be made of membranes stretched on a rigid frame. The alignment of the two reflectors is critical to the performance of the system, but an arrangement of six adjustable rigid struts connecting the two reflectors completely constrains all degrees of freedom while allowing the adjustment of the relative orientation of the two reflectors. Initial testing of a small prototype has been very encouraging ( 40 micron diffraction limit has been achieved), and a $2 \times 4 \mathrm{~m}$ model is now under test in a collaboration between JPL and Lockheed-Martin. The DART concept studied by the NEXT program produced a JPL Team-X study that included considerable thought about deployment, with and without astronauts.

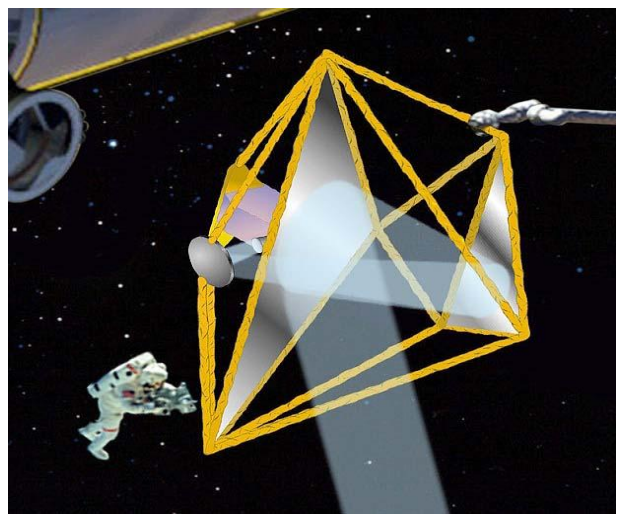

DART style SAFIR architecture. While especially ambitious for the near term and not as technically mature at the baseline design, DART offers a potentially economical pathway to huge light gathering powers for space submillimeter astronomy. While strategies for autonomous deployment are being developed, clear opportunities for astronaut assembly efforts were studied by the NASA Exploration Team, and found to provide value to the mission.

Our study includes a partnership with Lockheed-Martin, continuing previous test efforts (Tolomeo et al. 2003) to study critical technologies that need significant development. The study will include thermal control, gravity release effects, on-orbit figure adjustment and metrology, vibration isolation and pointing control. They will review techniques for optics membrane reflector deployment, and launch survivability, and explore options for 15-25 m aperture sizes.

Finally, we acknowledge the substantial heritage from the Large Deployable Reflector (LDR) effort of a decade past. Many of the technical hurdles that faced this observatory have been since eliminated. Interestingly, LDR evolved into an international mission that was then successfully scaled back in size to what is now Hershel, and our team is closely linked with that community in order to make sure that SAFIR has good scientific and technical ties.

We are paying special attention to optimal orbital venues. It is impossible to achieve the needed temperatures in LEO, so a prime target is Earth-Sun L2 - the location of WMAP and the destination for JWST. It is close enough for high RF telemetry rates (supporting anticipated $800 \mathrm{kbps}$ maximum using a daily one-hour DSN pass), and provides the needed shielding geometry. This orbit also allows a reasonably modest launch vehicle, given the baseline ( $\sim 5000 \mathrm{~kg})$ mass estimates. The Earth-trailing driftaway orbit of Spitzer avoids the need for station-keeping maneuvers (the L2 orbit is unstable), but requires more advanced telemetry for a long-lived mission. Telemetry may not be as serious an issue as for JWST, however, because of the lower number of pixels on board. Geosynchronous orbits would require much more capable sunshields and coolers by comparison. Orbits farther from the Sun would reduce the zodiacal light background and improve sensitivity, but may require special power sources for thermal management and communication.

The baseline configuration of the SAFIR calls for a roughly circular aperture shape, independent of mirror technology. This offers the most compact image, ultimate sensitivity for a fixed mirror area, and matches the typical square symmetry of detector arrays. However, at long wavelengths, image contrast may be more important than angular resolution. Resolution might be improved with non-circular symmetry, at cost of asymmetrical instantaneous images. Symmetrical images could be recovered by combining observations taken at many roll angles. We will search for an observatory configuration compatible with this mode, and will examine the scientific drivers for it. 
Every aspect of SAFIR must contribute to achieving an unprecedentedly large and cold telescope. The thermal design is thus the most important, and most complex, part. A successful thermal design must include the following key ingredients: maximal use of radiative cooling, efficient isolation, (both radiative and conductive, between warm and cold parts), and the use of active cryocoolers to supplement radiative cooling. Studies suggest that temperatures of $\sim 10 \mathrm{~K}$ may be achieved radiatively at L2. It can be seen from the plot below that a 4K SAFIR is background limited on the zodi and CMB. A central question in our mission concept study, therefore is: What are the limits of thermal isolation and radiative cooling, i.e. how low a temperature can be achieved passively? The answer will depend on the orbit, and on the structure and

Sparse aperture (non-circular) concept for SAFIR primary offers a larger maximum baseline, providing added spatial resolution. While conserving weight and total light gathering power, it would be achieved at the price of a non-circularly symmetric instantaneous diffraction spot.

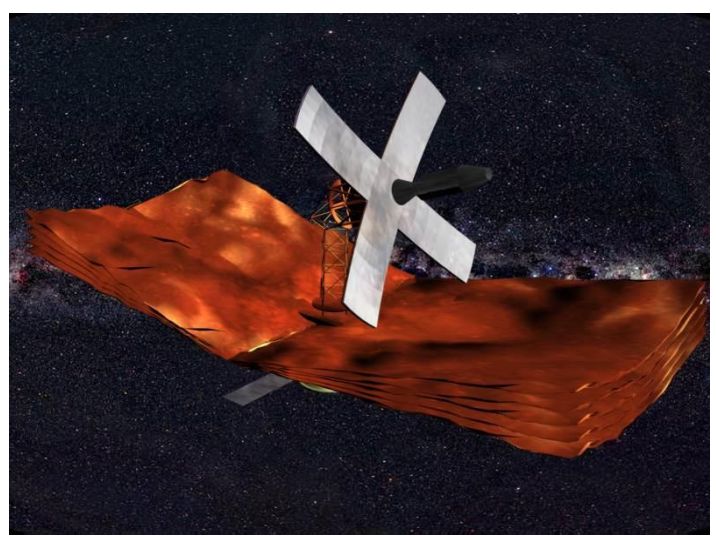

materials of SAFIR, which in turn depend on size and thermal shields. This will determine the amount of active cooling required, and the kind of active coolers that must be developed. A key tradeoff is between mirror diameter and temperature. The larger the mirror, the more photons are collected. But the dependence of SNR on mirror size depends on whether the dominant source of noise is from the detectors, telescope, diffuse sky backgrounds, or source photon statistics. Presently this tradeoff is hampered by our poor knowledge of the source density in the farIR. Fortunately, Spitzer will change this situation dramatically during the study. The number of design variables pertinent to the overall thermal design is huge, well beyond the possibility of an exhaustive search. As described above, we will use a different approach, starting with the architectures described above.

The SAFIR integration and test concepts will be developed based on those of the JWST and Spitzer missions. Ideally SAFIR will be tested on the ground as it will fly. Our study will consider infrastructure and constraints assumed in place at the time of implementation. The largest available test chamber is the Plumbrook facility at NASA's Glenn Research Center. Chamber modifications that would be required for SAFIR's lower temperature will be evaluated during this study. Changes may also be required in the methods used to compensate for gravity, since the larger SAFIR may be supported differently than JWST.

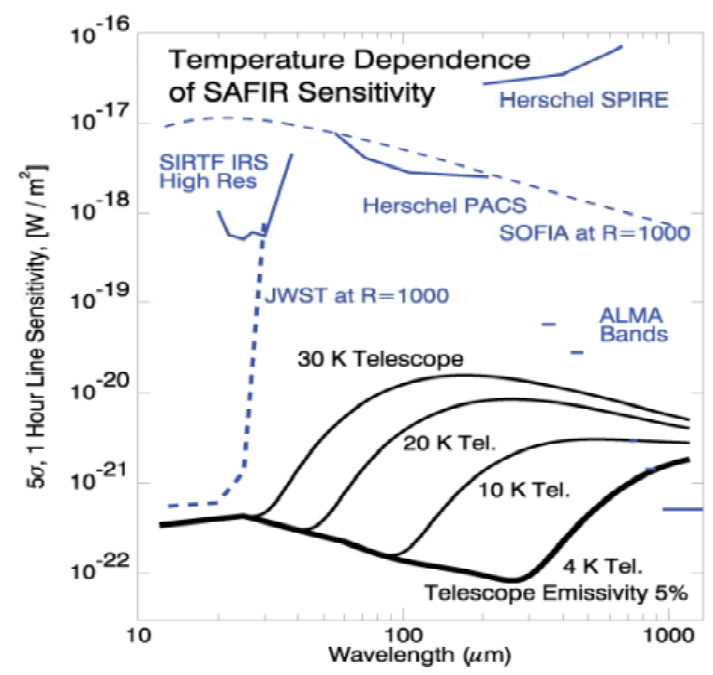


SAFIR's power system will be sized to meet observatory needs, which are dominated by the active cooling system. Cryocooler efficiency estimates lead to a $\sim 4 \mathrm{~kW}$ budget, which can be provided at L2 using solar panels of area $<20$ $\mathrm{m}^{2}$ mounted to the spacecraft bus on the sun-facing side. The design will be evaluated during the concept study as the cryocooler technology and thermal control design mature. Although nuclear power sources, both radioisotope thermal generators and space fission reactors, offer power that could provide truly impressive amounts of active cooling, these are not baselined for SAFIR. The waste heat from such reactors could, in fact, be a serious threat to the thermal performance of the observatory. While far infrared and submillimeter telescopes could see greatly reduced sky background from a site farther out in the solar system, the lower thermal load from direct sunlight offsets to some degree the power need for active cooling.

The SAFIR instrument complement with derive from the DRM, but it seems clear that in order to cover the wide range of wavelengths and spectral resolutions that drive the science, the telescope may need four instruments. A broadband camera with multiple bandpass filters, a low resolution $(\mathrm{R} \sim 100)$ spectrometers for shorter wavelengths that will target dust composition research, a moderate resolution spectrometer $(\mathrm{R} \sim 2000)$ for extragalactic star formation research, and a high resolution spectrometer $(\mathrm{R} \sim 100,000)$ for detailed studies of the gas phase ISM. The first two spectrometers would ideally be equipped for integral field spectroscopy. Our team is working closely with Ball Aerospace, which is considering system-wide strategies for accommodating these instruments in the SAFIR focal plane. Mass, volume, and power budgets are of special interest in this context.

\subsection{Enabling Technologies}

Based on work done to date, it is clear that a large, cold far-IR and submillimeter telescope can be flown in the next decade. The key task in our study will be to construct from among discrete possibilities an overall design concept that maximizes performance through innovation while minimizing cost and risk.

SAFIR places challenging demands on detector, cooled optic, sunshade and cryocooler technologies that must be carefully managed to achieve flight mature systems. Its unique capabilities will be realized at the cost of a deliberate and sustained investment. This is being pursued though both Code S and Code T, and in the case of the latter, special efforts are being made to explore the opportunities that the new exploration initiative can offer the mission. We are developing a SAFIR technology roadmap that will address the science driven needs of SAFIR with reasonable cost/schedule requirements. Although the science objectives are unique, there is significant technology synergy with other present and future code S missions such as TPF, JWST, and the Inflation Probe. This roadmap will explore these synergies and recommend an approach that will maximize the return on investments.

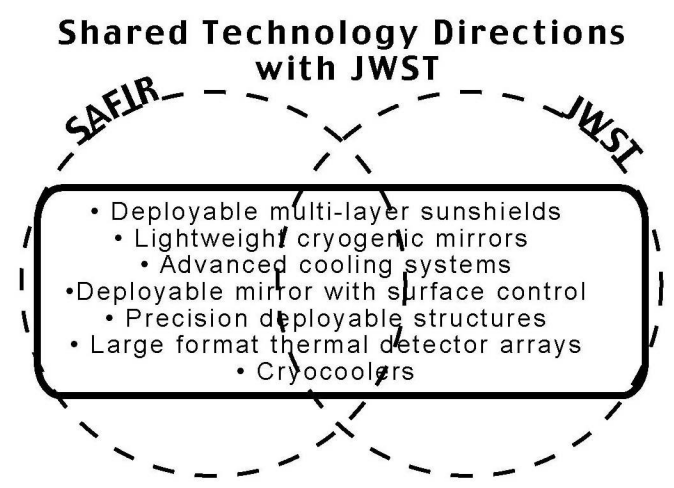

The drivers for a SAFIR technology roadmap are graphically outlined here. Shown above are technology needs that will benefit by strong heritage from JWST investments. In most of these technology cases, as well as I\&T, telescope pointing, alignment, spacecraft bus architecture, power, communication, and operations planning, present investments, including a wealth of cryogenic technology information that comes out of the Spitzer program bear directly on SAFIR planning.

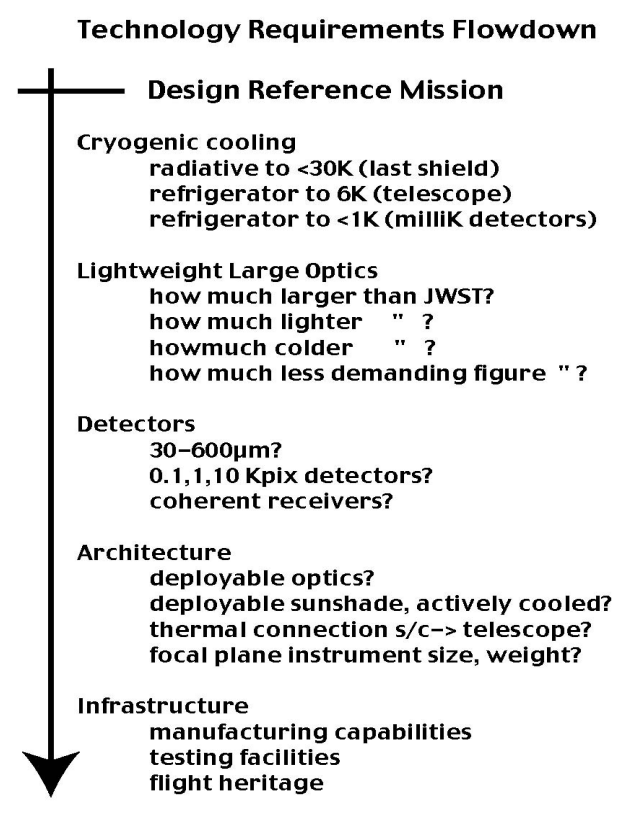


Detectors: The full promise SAFIR will only be realized if detectors of matching capabilities are available. SAFIR science will require significant effort in detector development, and an important effort under this proposal will be identification of detector needs. It has been well demonstrated that early investment in key technologies can pay large dividends in cost and success. The study will flow down the science requirements for SAFIR into detector technology needs. For example, in order to take advantage of the low background from SAFIR, detectors with NEPs less than a few $x 10^{-19} \mathrm{~W} \mathrm{~Hz}^{-1 / 2}$ will be needed for cameras, and lower for spectrometers.
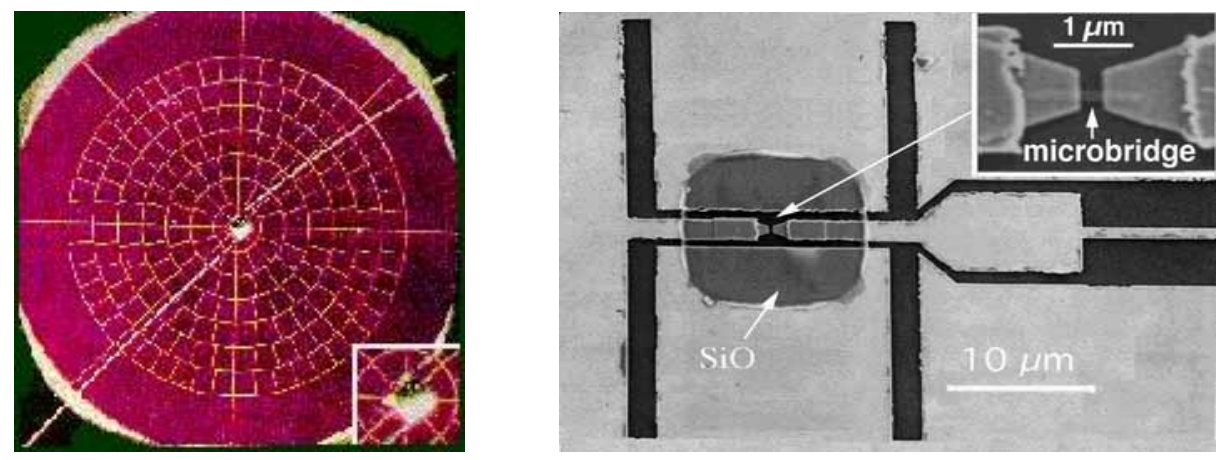

Large format infrared arrays (such as the SiN "spider web" bolometer design shown at left) promise to multiply the productivity of SAFIR. Especially for survey science, the productivity of the observatory is proportional to the number of detectors in the focal plane. Low noise oscillators and efficient mixers, shown at right, will provide new opportunities for high resolution studies with SAFIR. Focused technology investments will let us to realize these opportunities.

Both imaging and spectroscopic observations will require large, sensitive, and high dynamic range detector arrays. While remarkable progress has been made in producing very large arrays for $\lambda<40$ microns, significant effort will be needed to produce the desired systems for the far infrared and submillimeter. To support wide-field deep surveys or high resolution spectroscopy, arrays in excess of $10^{4}$ elements will be needed. Such element numbers have a large multiplicative effect on observatory productivity. The development of such large focal plane arrays in the far-IR and submillimeter bands represents a significant leap beyond the current state of the art.

The detector needs for these wavelengths have been addressed in OSS strategic planning efforts by the IR, Submm, and Millimeter Detector Working Group a number of the technologies, including superconducting and semiconducting bolometers and photoconductors, are being investigated under NASA funding. During this study we will assess them in the light of our concept and identify gaps.

Sunshades: Radiative cooling is a crucial part of SAFIR's thermal control. Sunshades use no power but intercept the majority of the thermal load. Optimized shielding of a Sun-illuminated observatory can reduce the thermal load by up to six orders of magnitude. We are investigating the optimization of multi-layer designs (including numbers of layers, layer spacing, and internal angles), deployability, and material properties. Thermal physics leads to a rapid drop of thermal radiator efficiency versus temperature, and the lowest achievable temperature can be affected by proper design. Proper design should allow the observatory to passively approach the ambient $7 \mathrm{~K}$ background that is found at Earth-Sun L2.For the lower temperatures that may be required, a particularly challenging facet of the baseline design is an actively cooled inner sunshade that would be kept at $15 \mathrm{~K}$. Our study will produce an estimate for the performance requirement for SAFIR's sunshield that will be used as an input to the upcoming Space Technology-9 in-space validation effort.

Cryocoolers: Optimized cryocoolers will be a critical part of both SAFIR and Life Finder type missions. They will be required not only for the sub-Kelvin focal planes supporting low noise detector operation, but also to achieve the low thermal background required by the science objectives. Our study is investigating unique requirements, their priority and sensitivity of design to each, and key technology risks and uncertainties. None of the cooling requirements for SAFIR is difficult to achieve with modest improvements on current technologies. The Advanced Cryocooler Technology Development Program (ACTDP) is taking a big step in this direction. These coolers are conservatively baselined as being able to lift $250 \mathrm{~mW}$ at $18 \mathrm{~K}$, and $7.5 \mathrm{~mW}$ at $6 \mathrm{~K}$ with flight-ready models available in 2005 - a full decade before launch of SAFIR. Early estimates suggest that about a half-dozen such coolers will allow the SAFIR telescope to be maintained at below $10 \mathrm{~K}$, and lower focal plane temperatures (down to $50 \mathrm{mK}$ ) can be maintained with several 
Continuous ADR coolers attached. The power budget for these appears to be manageable. Waste heat from these cryocoolers will be rejected by radiators mounted perpendicular to the Sun, but on the sunward side of the sunshield.

Cryogenic Optics: Our study will report on cryogenic mirrors, which are an enabling technology for SAFIR regardless of its eventual design configuration. Technical maturity of the process to manufacture and validate them and associated cost and schedule issues will be significant contributors to SAFIR's eventual flight architecture. Areal density $<10 \mathrm{~kg} / \mathrm{m}^{2}$, $<10 \mathrm{~K}$ temperatures, and $<1$ micron surface accuracy pose important technological challenges.

Our Technology Roadmap will include a review of mature cryogenic mirror technology in order to mitigate technical, cost and schedule risk early enough in the program to influence the architecture selection. We will take full advantage of the lessons learned for the JWST mirror concept development. After science requirements are translated into engineering specifications, our team will perform a technology gap analysis to assess the technical maturity of the engineering specifications. After an assessment of the current state-of-art and technology investments being made by all governmental agencies, our goal is to demonstrate SAFIR traceable mirrors at TRL-6 with attention to development risks and the difficult question of integration and test. For example, researchers at the Air Force Research Laboratory's Directed Energy directorate were recently able to produce a $1 \mathrm{~m}$ diameter mirror, made of a thin-film membrane material. This optical-quality polyamide mirror, about the thickness and flexibility of kitchen plastic wrap, was more than three times larger than the biggest membrane mirror previously possible. As noted above similar efforts are ongoing with DART. Our cryogenic mirror study plan will make maximum possible use of industry capabilities. New substrate materials such as $\mathrm{SiC}$ and $\mathrm{MgGr}$ will likely be considered as well as new fab processes such as replication. Membrane technologies will be evaluated as well. The ultimate goal is to minimize cost, schedule and technical risk for the manufacture, integration and test of SAFIR cryogenic optics.

\subsection{Deployment and In-Flight Validation}

Our study will report on deployment designs, including transportation to operational location and assembly as required. We will review designs developed for JWST and other missions to identify advantages or serious technology needs. Choices include the 3-leaf adopted for JWST, a flower petal design, various robotic assembly approaches that can pack many mirror segments into small volumes, the HARD (High Accuracy Reflector Deployment) method developed for other government agencies, and radically different methods suitable for the DART concept. If an inner sunshield with active cooling is required, special attention will need to be given to the flexible plumbing that would be required.

We will consider roles for astronauts and robots in assembly and on-orbit checkout. On the basis of visionary plans by the NEXT team, such roles may minimize risk and be highly enabling even though the premise of the baseline SAFIR plan does not require them. By emphasizing assembly, rather than autonomous deployment, it is an approach that expands the trade space for structural geometry, and can lead to higher performance for lower total mass. In this context, as well as others (e.g. I\&T, assurance, maintenance, operations servicing), our proposal includes a effort by Boeing to map human in-space capabilities onto SAFIR-specific needs. This will identify the technology required to support human presence and participation, cataloging the pros and cons of human participation, as well as a ROM cost difference between human-assisted and autonomous options. It will consider the complementary role played by robotic assistants, to optimize the mix of robots and humans in task performance. The capabilities of the CEV, and it's value to SAFIR for deployment, on-orbit testing, and servicing, will be considered. Our study team will ensure consistency of possible astronaut roles for SAFIR with the direction of the Space Architects Office, and the new President's Space Initiative.

\subsection{Operations}

A JWST-like operations scenario is envisaged for SAFIR, an assumption that we will evaluate against the DRM. The instrument complement and the importance of survey-mode observations will be key factors. This will include consideration of FOV constraints that are driven by Sun angle and concomitant thermal control. The study will consider the level of autonomy that the spacecraft will require, determined in part by the bandwidth of the communication segment, though the space segment can be largely autonomous after the commissioning period.

\section{SUMMARY}

The Vision Mission study for SAFIR is now underway, a study that will refine the science case and do a detailed summary evaluation of the technical readiness for the mission. The goal of this study is to prioritize the science drivers 
for the mission, and develop a design reference mission that is responsive to them. This DRM will provide pointers to optimal architectures, and provide important guidance about requirements, in particular for observatory temperature and instrument complement. Capitalizing on the engineering being developed for JWST, dramatic increases in infrared sensor sensitivity and format size, infrared science advances from Spitzer (and soon, SOFIA and Herschel), SAFIR is an observatory that has strong drivers. It lays the science and technological groundwork for more ambitious missions farther in the future, and addresses the strategic goals of the astronomy community.

\section{REFERENCES}

Amato, M.J., Benford, D.J., Moseley, S.H., and Roman, J. An Engineering Concept and Enabling Technologies for a Large Single-Aperture Far-IR Observatory (SAFIR), 2003 Proc. of SPIE, Vol 4850, p. 1120.

Dragovan, M., DART System for FarIR Applications, 2003 Proc. of SPIE, Vol 4849, p. 8.

Tolomeo, J. et al., Design and Test of a 2mx4m DART System in the RF, 2003 Proc. of SPIE, Vol 5166, p.124.

Soifer, B.T., and Pipher, J.L., Instrumentation for Infrared Astronomy, 1978 Annual Reviews of Astronomy and Astrophysics, 16, 335, 1978.

Beichman, C.A. et al. Infrared Astronomical Satellite (IRAS): v. 1 - Explanatory Supplement, NASA, Washington DC 1988.

Kessler M.F., et al., The Infrared Space Observatory (ISO) mission, 1996 Astronomy and Astrophysics 315, L27.

Gallagher, D., Irace, W., and Werner, M., Development of the Space Infrared Telescope Facility, 2002 Proc. of SPIE, Vol 4850, p. 17. 